\title{
A Magnetic Field Sensor Based on a Magnetic Fluid-Filled FP-FBG Structure
}

\author{
Ji Xia ${ }^{1}$, Fuyin Wang ${ }^{1}$, Hong Luo ${ }^{1}$, Qi Wang ${ }^{2}$ and Shuidong Xiong ${ }^{1, *}$ \\ 1 Academy of Ocean Science and Engineering \& College of Optoelectronic Science and Engineering, \\ National University of Defense Technology, Changsha 410073, China; blovexiaji@163.com (J.X.); \\ fuyin_wang@126.com (F.W.); luohongrong2000@163.com (H.L.) \\ 2 College of Information Science and Engineering, Northeastern University, Shenyang 110819, China; \\ wangqi@ise.neu.edu.cn \\ * Correspondence: nudtxsd@163.com; Tel.: +86-138-0847-6806
}

Academic Editor: Vittorio M. N. Passaro

Received: 3 March 2016; Accepted: 21 April 2016; Published: 29 April 2016

\begin{abstract}
Based on the characteristic magnetic-controlled refractive index property, in this paper, a magnetic fluid is used as a sensitive medium to detect the magnetic field in the fiber optic Fabry-Perot (FP) cavity. The temperature compensation in fiber Fabry-Perot magnetic sensor is demonstrated and achieved. The refractive index of the magnetic fluid varies with the applied magnetic field and external temperature, and a cross-sensitivity effect of the temperature and magnetic field occurs in the Fabry-Perot magnetic sensor and the accuracy of magnetic field measurements is affected by the thermal effect. In order to overcome this problem, we propose a modified sensor structure. With a fiber Bragg grating (FBG) written in the insert fiber end of the Fabry-Perot cavity, the FBG acts as a temperature compensation unit for the magnetic field measurement and it provides an effective solution to the cross-sensitivity effect. The experimental results show that the sensitivity of magnetic field detection improves from $0.23 \mathrm{~nm} / \mathrm{mT}$ to $0.53 \mathrm{~nm} / \mathrm{mT}$, and the magnetic field measurement resolution finally reaches $37.7 \mathrm{~T}$. The temperature-compensated FP-FBG magnetic sensor has obvious advantages of small volume and high sensitivity, and it has a good prospect in applications in the power industry and national defense technology areas.
\end{abstract}

Keywords: magnetic field sensor; Fabry-Perot Cavity; magnetic fluid; Fiber Bragg Grating; temperature compensation

\section{Introduction}

Magnetic field sensors have been widely applied for navigation, vehicle detection, current sensing, and spatial and geophysical researches. Compared with the other types of magnetic field sensor, all-fiber based sensors have been extensively investigated owing to their portability, high geometric adaptability, immunity from electromagnetic interference, long distance signal transmission for remote operation, and resistance to high pressure and corrosion.

Magnetic fluids (MFs) have numerous interesting optical characteristics [1-3], such as tunable refractive index, tunable transmittance, birefringence and thermal lens effects, etc. Generally, the components of a MF are magnetic particles, a carrier liquid and surfactants. When an external magnetic field or thermal effect is applied to a MF, then the refractive index of the MF varies with the changing magnetic field or thermal effect. Various MF-based optical devices have been developed [4-6], such as magnetic fluid light modulators, magnetic fluid optical gratings, and magnetic fluid optical fiber filters, etc.

Recently, various optical fiber structures with MFs have been proposed for magnetic field sensing, The employed structures include Fabry-Perot cavity [7,8], polymer optical fiber [9], 
fiber Bragg grating [10,11] multimode interference [12,13] Michelson interferometer [14] Sagnac interferometer [15,16] and photonic crystal fiber (PCF)-based interferometers [17-19]. Homa [7] used a MF-filled extrinsic FP interrogated with an infrared wavelength spectrometer to detect magnetic fields. The sensor readily measured the magnetic field with a range of $0.5 \mathrm{mT}$ to $12.0 \mathrm{mT}$ with corresponding sensitivities in the 0.3 to $2.3 \mathrm{~nm} / \mathrm{mT}$ range. In 2014, Lv [20] proposed a novel fiber-optic magnetic field sensor, which was composed of an extrinsic fiber Fabry-Perot interferometer and a magnetic fluid. Preliminary experiments illustrated that the magnetic field measurement sensitivity was $0.0431 \mathrm{~nm} / \mathrm{Gs}$ and the measurement resolution was better than $0.5 \mathrm{Gs}$ in the range from 0 to $400 \mathrm{Gs}$. Their work however did not take the thermal effect of the MF into consideration. The refractive index of the MF behaves differently under varying magnetic field and temperature conditions, which causes a cross-sensitivity effect from the temperature and magnetic field existing in the MF. The measurement systems mentioned above don't take the impact of the operating temperature on the characteristic refractive index into account, which makes the resulting magnetic field measurements unstable and inaccurate. In this work, a modified sensor probe based on the multiplex FP-FBG structure is put forward for temperature compensation, which solves the cross-sensitivity effect of the temperature and magnetic field in the MF. The FBG is written on the insert fiber end of the FP cavity, and it is sensitive to the temperature variation but insensitive to magnetic field changes. The resulting FP-FBG structure is experimentally demonstrated for high resolution magnetic field detection.

\section{Principles of the FP-FBG Sensor Filled with Magnetic Fluid}

The multiplexing structure based on the FP-FBG sensor is shown in Figure 1, where the FBG is written on the insert fiber end of the FP cavity. When a broadband light beam with light intensity Iin transmits through the FBG, the reflected light intensity near the Bragg reflection wavelength is I1. Then the transmission light beam intensity I2 of FBG reaches the FP cavity. In a low-reflectivity FP cavity, the interference spectrum intensity of $\mathrm{FP}_{3}$ is generated and transmitted back through the FBG once again. Finally, the reflected light beam intensity $\mathrm{I}_{\text {out }}$ from the FP-FBG sensor is combined with the second FBG transmission light beam intensity $\mathrm{I}_{4}$ and the former FBG reflected light beam intensity $\mathrm{I}_{1}$, and the output light intensity of the FP-FBG sensor $\mathrm{I}_{\text {out }}$ can be obtained as below:

$$
I_{\text {out }}=I_{1}+I_{4}=I_{\text {in }} \cdot\left[f_{F B G}+\left(1-f_{F B G}\right)^{2} \cdot f_{F-P}\right]
$$

where, $f_{F B G}$ is the reflection coefficient of FBG and it is defined as $f_{F B G}=R \cdot \exp \left[-\left(\lambda-\lambda_{B}\right)^{2} / c^{2}\right], R,, c$ are the Bragg peak reflectivity, the center-reflected wavelength of FBG, and the bandwidth of the FBG reflected peaks. Accordingly, $f_{F-P}=2 r \cdot[1+\cos (4 \pi L / \lambda+\pi)]$ is the reflection coefficient of FP cavity, and $r$ is the reflectivity of fiber end, $L$ is the length of FP cavity.

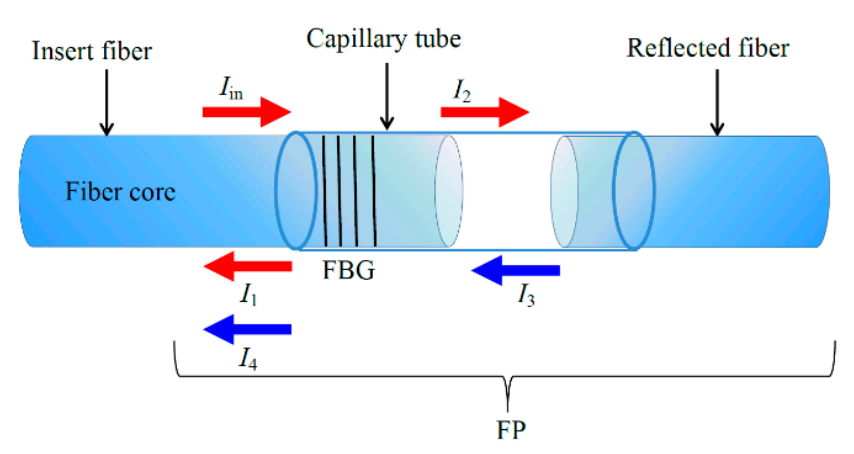

Figure 1. The structure of a FP-FBG sensor.

Meanwhile, the resonant peak wavelengths of FBG and FP should be set within a proper measurement wavelength-range from the input light and both of them can be distinguished through one another. In order to maintain the FP interference fringes and the FBG reflected peaks at a same 
order of magnitude, the reflectivity of FBG should be set to close to that of the low fitness FP sensor. Figure 2 shows the reflected spectrum simulation of the FP-FBG sensor.

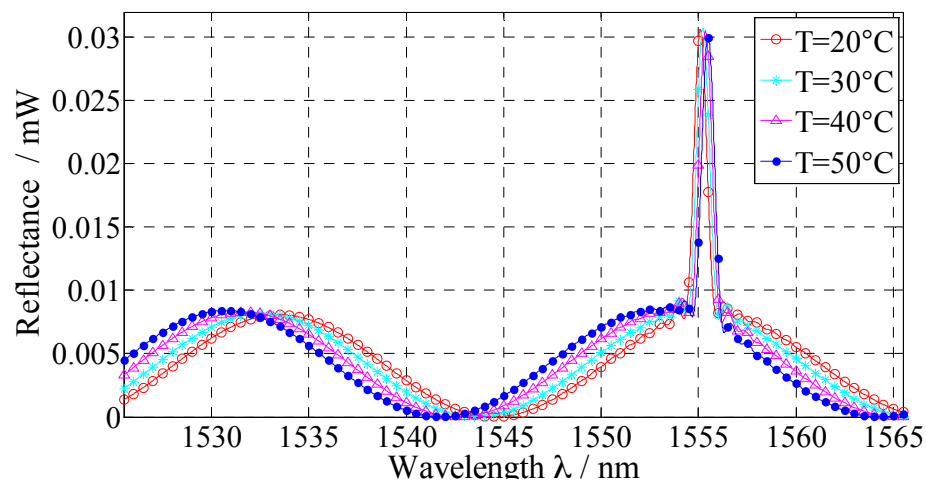

Figure 2. Simulation of the output spectrum under different temperatures $(\mathrm{H}=0)$.

In the simulation, the length of FP cavity is set as $L=40 \mu \mathrm{m}$, the center-reflected wavelength of FBG at room temperature $25^{\circ} \mathrm{C}$ is $\lambda_{B}=1550 \mathrm{~nm}$ with a reflectivity of $4 \%$. The intensity of the incident light is $1 \mathrm{~mW}$ with a spectrum range of $1525 \mathrm{~nm}-1565 \mathrm{~nm}$. The simulated spectrum of the FP-FBG is displayed clearly with combination of two reflected spectra at a same spectral resolution and the reflected spectra moves through one another without interference. As the temperature increases from $20{ }^{\circ} \mathrm{C}$ to $50{ }^{\circ} \mathrm{C}$, the interference spectrum of the FP sensor shifts towards the short wavelength direction, however, the center-reflecting wavelength of the FBG moves towards along the long wavelength direction. In Figure 2, the amount of movement of the FBG resonant peaks is less than that of the FP interference fringes.

With reference to the research results of Chen et al. [21], under a constant temperature value of $T$, the relationship between the refractive index of the MF and the magnetic field is as below:

$$
n_{M F}=\left(n_{s}-n_{0}\right)\left[\operatorname{coth}\left(\alpha \frac{H-H c}{T}\right)-\frac{1}{\alpha\left(H-H_{c}\right)}\right]+n_{0}
$$

where, $H c$ is the critical value of the applied magnetic field $(H>H c), n_{0}$ is the refractive index of the MF with the critical magnetic field and $n_{\mathrm{S}}$ is the saturated refractive index of MF, and $\alpha$ is a fitting coefficient. Generally, the parameters of $n_{\mathrm{s}}, n_{0}, \alpha, H c$ for a certain kind of magnetic fluid film are regarded as constants. Therefore, at a certain experimental temperature $T$, there is only one refractive index for the MF $n_{\mathrm{MF}}$ for a certain magnetic field $H$. The refractive index of the MF $n_{\mathrm{MF}}$ is tested under different magnetic field and temperature conditions, and the experimental results are used for magnetic field detection with temperature compensation.

In the sensor probe with a FP-FBG structure, the FBG is written on the insert fiber end of the FP cavity. Being inspired by the concept that the central wavelength shifts of the FBG are applied to detect the external varying temperature, the central wavelength shift of FBG can be defined by Equation (3):

$$
\Delta \lambda_{B}=\lambda_{B}\left(\beta_{T n}+\beta_{T l}\right) \Delta T
$$

where, $\beta_{T n}, \beta_{T l}$ are the thermal-optical effect coefficient of $8.0 \times 10^{-6} /{ }^{\circ} \mathrm{C}$ and thermal expansion effect coefficient of $0.55 \times 10^{-6} /{ }^{\circ} \mathrm{C}$ in the FBG. $\Delta T$ is the variation value of the operating temperature. It is noted that the central wavelength shift of FBG is not sensitive to the magnetic field applied on the FP-FBG sensor, which is used for the measurement of magnetic field with temperature compensation. Due to the cross-sensitivity effect of the temperature and magnetic field in MF, the characteristic magnetic fluid refractive index are defined as follows:

$$
\Delta n_{M F}=\alpha_{H n} \cdot \Delta H+\alpha_{T n} \cdot \Delta T
$$


where $\Delta n_{M F}$ is the change of the magnetic fluid refractive index, and $\alpha_{H n}, \alpha_{T n}$ are the magnetic field sensitive coefficient and temperature sensitive coefficient of the magnetic fluid, respectively. Considering the thermal expansion effect of optical fiber, the central wavelength shift in the output spectrum of the FP-FBG magnetic sensor is given by Equation (5):

$$
\Delta \lambda_{m}=\lambda_{m}\left[\alpha_{H n} \cdot \Delta H+\left(\alpha_{T n}+\alpha_{T l}\right) \cdot \Delta T\right]
$$

where, $\Delta \lambda_{m}$ is the central wavelength shift of the FP-FBG magnetic sensor, and $\alpha_{T l}$ is the optical fiber thermal expansion effect coefficient. Combining Equations (3) and (5), a relationship matrix between the FBG output wavelength shifts and the two under-test parameters is obtained in Equation (6). Equation (7) achieves the measurement of the temperature and magnetic field through calculating the matrix in Equation (6):

$$
\begin{gathered}
{\left[\begin{array}{c}
\Delta \lambda_{m} \\
\Delta \lambda_{B}
\end{array}\right]=\left[\begin{array}{cc}
\alpha_{H n} \lambda_{m} & \left(\alpha_{T n}+\alpha_{T l}\right) \lambda_{m} \\
0 & \left(\beta_{T n}+\beta_{T l}\right) \lambda_{B}
\end{array}\right]\left[\begin{array}{c}
\Delta H \\
\Delta T
\end{array}\right]} \\
{\left[\begin{array}{c}
\Delta H \\
\Delta T
\end{array}\right]=\left[\begin{array}{cc}
\alpha_{H n} \lambda_{m} & \left(\alpha_{T n}+\alpha_{T l}\right) \lambda_{m} \\
0 & \left(\beta_{T n}+\beta_{T l}\right) \lambda_{B}
\end{array}\right]^{-1}\left[\begin{array}{c}
\Delta \lambda_{m} \\
\Delta \lambda_{B}
\end{array}\right]}
\end{gathered}
$$

Obviously, when the spectrum drifts and the temperature detected by the FBG are measured in an experimental system, then the change of the magnetic field $\Delta H$ can be obtained by the matrix in Equation (7). According to Equation (7), the MF-filled FP-FBG sensor can achieve the simultaneous measurement of the magnetic field and the temperature, and the temperature detected in real-time can be used for the compensation of the magnetic field measurement. Finally, the cross-sensitivity effect of the temperature and magnetic field in the magnetic fluid is eliminated.

\section{Experiment System and Analysis}

The magnetic field measurement system setup is shown in Figure 3. The light beam output from a $1550 \mathrm{~nm}$ DFB is injected into the optical fiber circulator via port1, and it transmits into the MF-filled FP-FBG sensor for magnetic field and temperature detection. The programmable DC controls the power of the output electric current to generate a stable magnetic field through a set of coils. When the applied magnetic field varies, the refractive index of the MF changes and the light signal is modulated. The reflected light passes through port 2 to port 3 and it is detected by the spectrometer (AQ6370 OSA, YOKOGAWA, Tokyo, Japan, with a wavelength resolution of $0.02 \mathrm{~nm}$ ). The MF-filled FP-FBG sensor is located in a temperature control oven (FLYSET Temperature Controller, Shenzhen Dragon Born Hot Runner Technology Co., LTD, Shenzhen, China, with a temperature resolution of $\pm 1^{\circ} \mathrm{C}$ ) for the tests at different stable temperatures, and the thermometer is put close to the sensor head to detect the operating temperature. In addition, a Gauss meter is also located near the sensor head to measure the varying magnetic fields. The temperature variation detected by thermometer and the magnetic field variation detected by the Gauss meter are taken to compare with the measurement results of the MF-filled FP-FBG sensor. As shown in the inset of Figure 3, the MF-filled FP-FBG sensor has a FBG length of $10 \mathrm{~mm}$ with a refractive index of $\mathrm{R}=4 \%$ (the grating wavelength is $1550 \mathrm{~nm}$ ). The length of MF filled in the FP cavity is fabricated with $32 \mu \mathrm{m}$. First, the fiber end written with a FBG inserts into the capillary tube; after the fiber is aligned with the capillary it is observed under a microscope; the MF (EMG605, Ferrotec USA Corporation, Bedford, NH, USA) with a volume content of C=1.8\% is infiltrated into the capillary tube. Finally, the reflected fiber is inserted into the capillary tube and sealed with UV glue under ultraviolet light irradiation for $24 \mathrm{~h}$. 


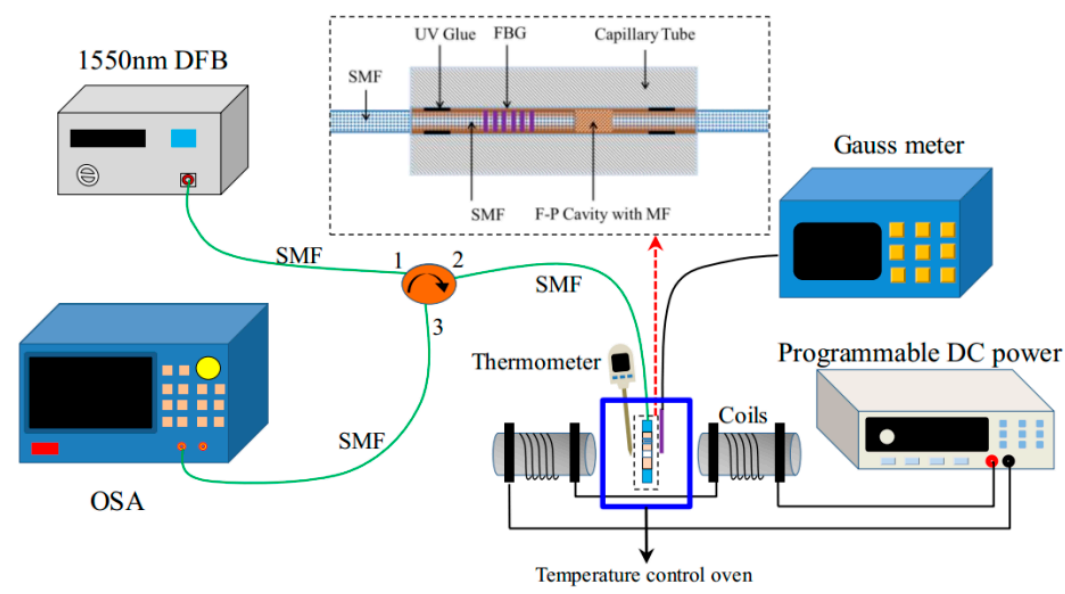

Figure 3. Experimental setup configuration for the FP-FBG sensing system. Inset: the cross-section schematic diagram of the FP sensing head.

To illustrate the operation, the optical fiber end face reflection method based on the Fresnel reflection principle is performed to measure the characteristics of refractive index of the MF under different magnetic fields and temperatures, as shown in Figure 3. The refractive index of the MF under parallel magnetic fields behaves as shown in Figure $4 \mathrm{a}$, which shows that $n_{\mathrm{MF}}=1.3414$ without any applied magnetic field.

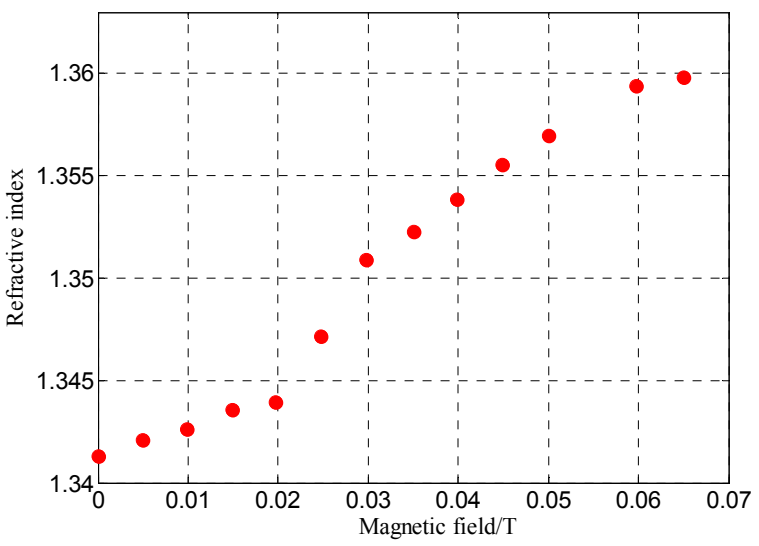

(a)

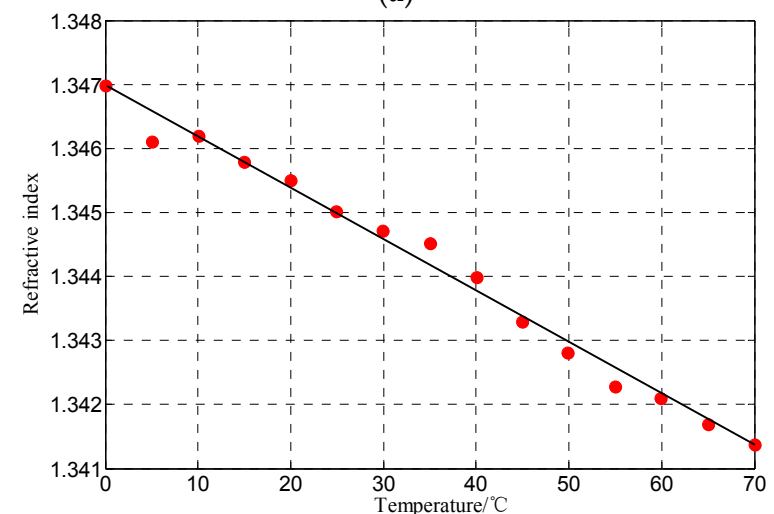

(b)

Figure 4. (a) Magnetic fluid refractive index variation versus transverse magnetic field; (b) Magnetic fluid refractive index variation versus temperature. 
When $H$ is less than $20 \mathrm{mT}, n_{\mathrm{MF}}$ increases gradually while the variation is very little. With the $H$ increases from $20 \mathrm{mT}$ to $60 \mathrm{mT}$, the corresponding $n_{\mathrm{MF}}$ increases from 1.3446 to 1.3600 with high sensitivity, which plays an important role in determining the effective operating magnetic field range in the experiment. Although $H$ continues to increase from $60 \mathrm{mT}, n_{\mathrm{MF}}$ does not change obviously, which indicates that $n_{\mathrm{MF}}$ has reached its saturation value under the strong magnetic field. Therefore, the MF has an effective working magnetic field range in the experiment, and the range of magnetic field $H$ is set from $0 \mathrm{mT}$ to $40 \mathrm{mT}$. When the direction of magnetic field is transverse to the direction of the incident light, and the polarizability of MF increases with the increase of the magnetic field (magneto-electric effect). The change of $n_{\mathrm{MF}}$ is similar to the performance of MF polarizability, and it is dependent on the counter-direction between the applied magnetic field and the light transmitting through the MF. When the magnetic field is set as $0 \mathrm{~T}$, the refractive index of MF is shown in Figure $4 \mathrm{~b}$. As the temperature varies from $0{ }^{\circ} \mathrm{C}$ to $70^{\circ} \mathrm{C}$, the refractive index of the MF decreases linearly with a temperature sensitivity of $-0.00008 \mathrm{RIU} /{ }^{\circ} \mathrm{C}$. When $\mathrm{MF}$ is simultaneously subjected to an operating magnetic field and a changing temperature, the refractive index of the MF behaves according to both of them, making it hard to determine the refractive index of the MF as it responds to the the changing magnetic field or changing temperature and this will lead to an inaccurate magnetic field detection. Hence, it is necessary to take effective measures to compensate the operating temperature in the magnetic field measurement. With $\mathrm{PW}^{*} @$ work the temperature in the experiment system is set to $25^{\circ} \mathrm{C}$, and the FP-FBG sensor filled with MF is located in a temperature control oven between a set of coils. The magnetic field direction is transverse to that of the incident light. As the applied magnetic field controlled by the programmable DC power increases from $0 \mathrm{mT}$ to $39.12 \mathrm{mT}$, the normalized interference spectrum of the FP-FBG sensor shift towards the long wavelength direction (near the wavelength of $1550 \mathrm{~nm}$ ) as shown in Figure 5.

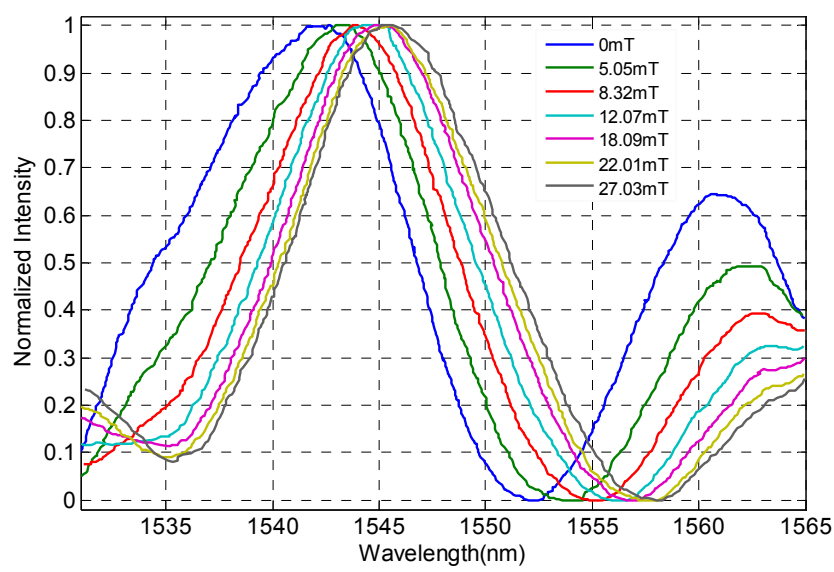

Figure 5. Spectrograms under a magnetic field range of $0 \sim 30 \mathrm{mT}$.

Figure 6 gives the FP-FBG sensor test results under two magnetic fields. At room temperature $\left(25^{\circ} \mathrm{C}\right)$ it can be obtained that the interference fringes of FP move with the magnetic field variation but the reflected peak of the FBG is unchanged.

The result in Figure 6 is in good agreement with the conclusion that the FBG written in the insert end of FP cavity is insensitive to magnetic field changes. The tests (the test was repeated three times) for a MF-based FP-FBG sensor under different magnetic fields are shown in Figure 7, where the curve fitting of the relationship between resonance peak drifts and the magnetic field indicates the MF-based FP-FBG sensor has a magnetic field sensitivity of $0.34 \mathrm{~nm} / \mathrm{mT}$ with a repeatability error of $R^{2}=1.5 \%$.

The interference spectrum of the MF-filled FP-FBG sensor which shifts under different temperature behaves as seen in Figures 8 and 9 . 


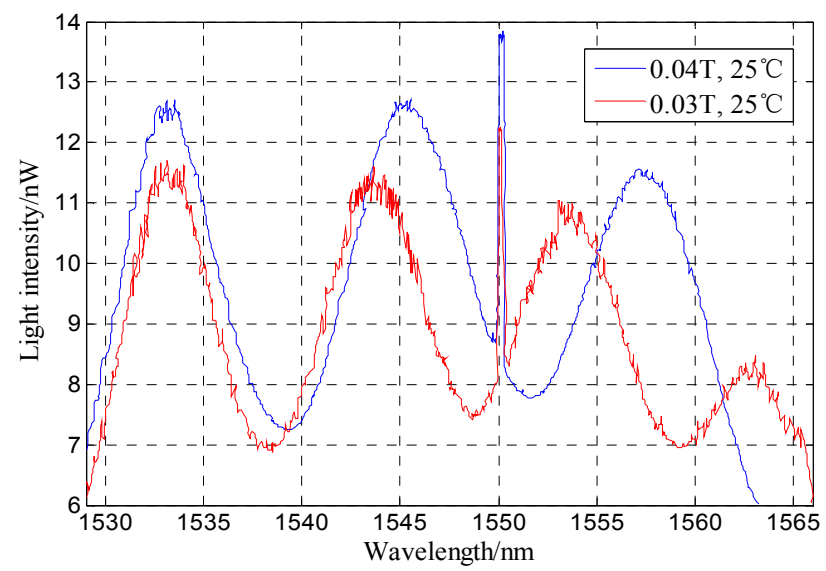

Figure 6. Spectrograms under a magnetic field range of $0 \sim 30 \mathrm{mT}$.

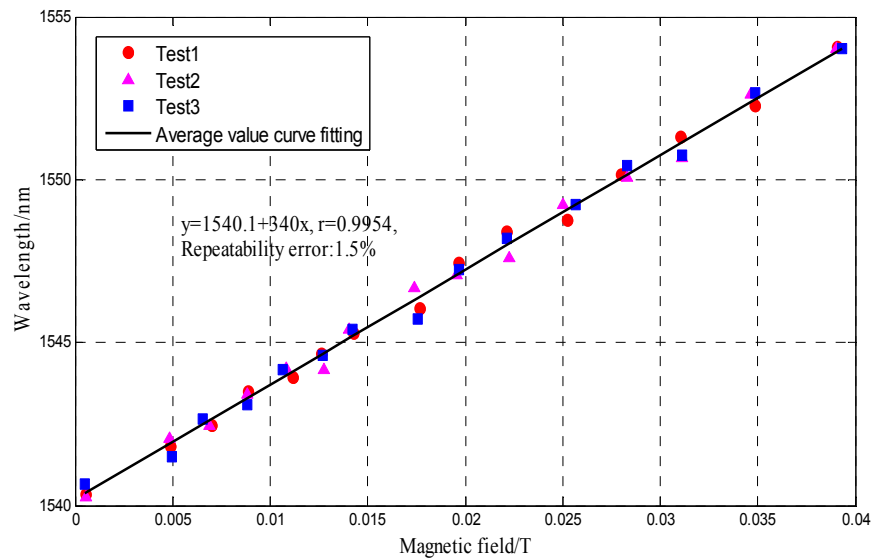

Figure 7. FP peak wavelength variation versus magnetic field.

Considering that the output spectrum of the MF-filled FP-FBG sensor is combined with the reflected spectra of the FP sensor and FBG sensor and their resonance peak shifts, the analysis of the interference spectrum of FP-FBG sensor under different temperatures can be equally separated into those of the FP sensor and FBG sensor. As the temperature increases, the refractive index of the MF decreases and the output interference spectrum of the FP sensor shifts in a shorter wavelength direction. When the resonance peak of the FP sensor moves within the temperature range $20{ }^{\circ} \mathrm{C}<\mathrm{T}<$ $95^{\circ} \mathrm{C}$, the MF-filled FP-FBG sensor has a temperature sensitivity of $0.092 \mathrm{~nm} /{ }^{\circ} \mathrm{C}$ with a repeatability error $R^{2}=0.8 \%$. As illustrated in Figure 9, the center wavelength of the FBG sensor shifts less than that of the FP sensor, and the FBG has a temperature sensitivity of $0.013 \mathrm{~nm} /{ }^{\circ} \mathrm{C}$ with a repeatability error $R^{2}=1.2 \%$.

Finally, when the magnetic field and temperature are applied to the MF-filled FP-FBG sensor, the corresponding magnetic field and temperature can be calculated from Equation (7) combined with the analysis of Figures 7-9. In the system, the temperature measurement can be used as a compensation for the magnetic field detection by the FP-FBG sensor filled with MF. According to Equation (7), a magnetic field sensitivity of $0.34 \mathrm{~nm} / \mathrm{mT}$, temperature sensitivity of $0.092 \mathrm{~nm} /{ }^{\circ} \mathrm{C}$ in the MF-filled FP sensor and a temperature sensitivity of $0.013 \mathrm{~nm} /{ }^{\circ} \mathrm{C}$ in the FBG sensor can be obtained:

$$
\left[\begin{array}{c}
\Delta H \\
\Delta T
\end{array}\right]=\left[\begin{array}{cc}
0.34 \mathrm{~nm} / \mathrm{mT} & -0.092 \mathrm{~nm} /{ }^{\circ} \mathrm{C} \\
0 & 0.013 \mathrm{~nm} /{ }^{\circ} \mathrm{C}
\end{array}\right]^{-1}\left[\begin{array}{c}
\Delta \lambda_{m} \\
\Delta \lambda_{B}
\end{array}\right]
$$


Based on the sensing characteristic matrix in Equation (8) of the FP-FBG magnetic sensor, the MF-filled FP-FBG sensor (after compensation) and the MF-filled FP sensor (before compensation) are simultaneously tested in the magnetic field range $0.02 \mathrm{~T}<\mathrm{H}<0.06 \mathrm{~T}$. First, the temperature in the sensor head area is accurately measured by the reflected peak wavelength of the FBG; second, the wavelength of the FP-FBG varying with the magnetic field is modified according to the sensing characteristic matrix in Equation (8) and the magnetic field with temperature compensation is obtained in Figure 10. Figure 10 illustrates the fitted curves based on the test points. The magnetic field sensitivity after compensation is improved to $0.53 \mathrm{~nm} / \mathrm{mT}$ with the comparison of the magnetic field sensitivity before compensation of $0.23 \mathrm{~nm} / \mathrm{mT}$. As the wavelength measurement resolution of OSA is $20 \mathrm{pm}$, the magnetic field measurement resolution of FP-FBG sensor could reach $37.7 \mu \mathrm{T}$.

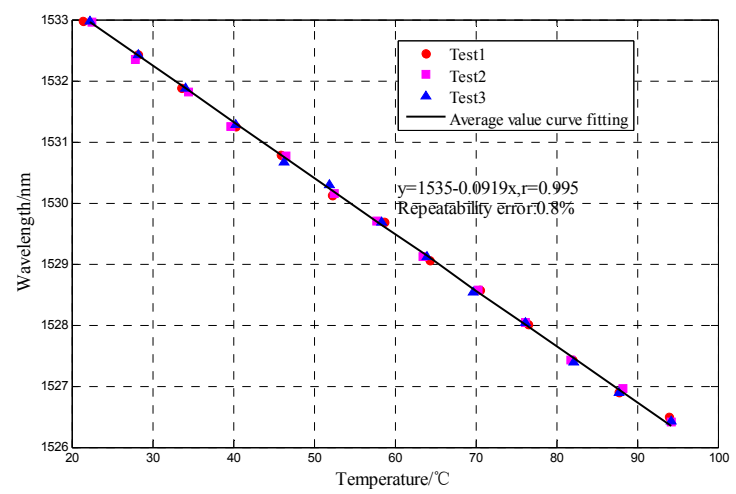

Figure 8. FP peak wavelength shift variation versus temperature.

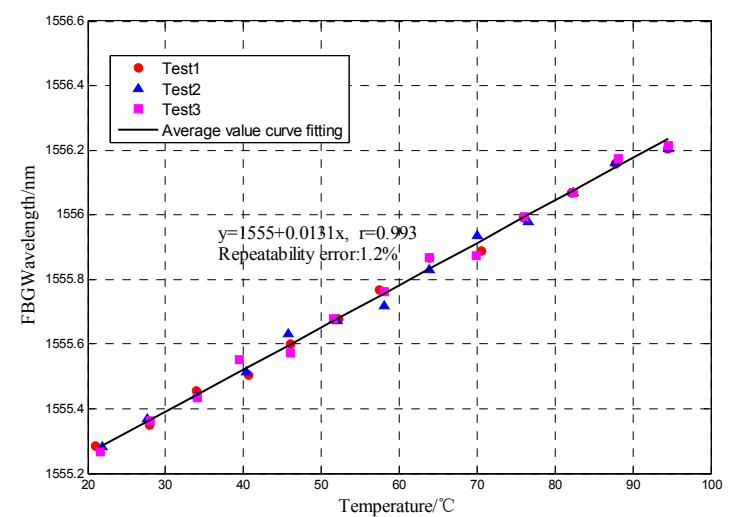

Figure 9. FBG wavelength shift variation versus temperature.

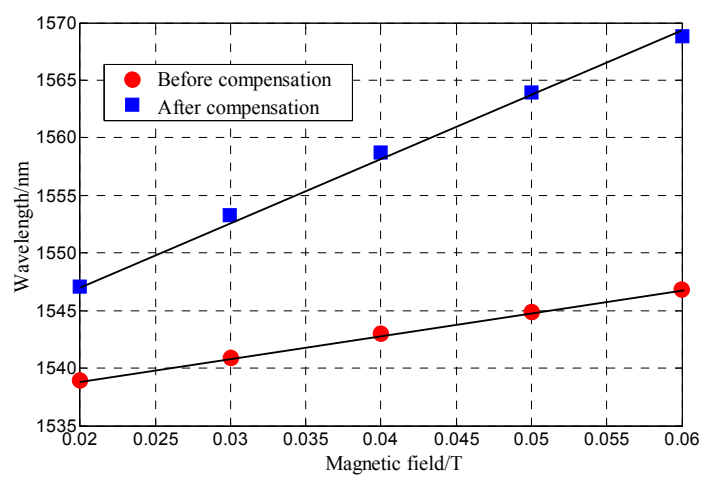

Figure 10. The test comparison of magnetic field sensitivity in the MF-filled FP-FBG sensor. 


\section{Conclusions}

Based on the characteristics of the refractive index of a MF, a fiber optic FP-FBG magnetic field sensor is proposed and demonstrated for magnetic field measurements with temperature compensation. The key point in the magnetic field measurement is to overcome the cross-sensitivity effect of the temperature and magnetic field in the MF. The FBG is written on the insert fiber of FP cavity for the operating temperature detection. Due to the fact the FBG is sensitive to temperature variations, the operating temperature in the sensing area can be measured and compensated for the magnetic field measurement. Preliminary experimental results show that the sensitivity of magnetic field measurement could reach $0.53 \mathrm{~nm} / \mathrm{mT}$ and the magnetic field measurement resolution could reach $37.7 \mu \mathrm{T}$. The FP-FBG magnetic field sensor probe has the advantages of simple structure, easy fabrication, anti-corrosion properties, low cost, and so on, and this sensor would find potential applications in the measurement of electromagnetic fields.

Acknowledgments: This work was supported by the National High Technology Research and Development Program of China (863 Program) under Grant 2013AA09A412-1, the Major Application Basic Research Project of NUDT under Grant number ZDYYJCYJ20140701, the National Natural Science Foundation of China under Grant 61203206, the Specialized Research Fund for the Doctoral Program of Higher Education of China under Grant 20120042120038.

Author Contributions: J.X. has developed the EF-DCM algorithm, conceived and designed the MF-filled FP-FBG sensor experiments and drafted the paper. And he played an important role in interpreting the results. F.W. has performed the experiments, contributed experiment materials and analysis tools, and contributed significantly to acquisition of data, analysis and interpretation of data. Both J.X. and F.W. have contributed equally to this work. H.L. and S.X. has contributed to the conception of the study, and helping perform the analysis with constructive discussions. Q.W. and S.X. has contributed to manuscript preparation, revising it critically for important intellectual detail and the final approval of the version to be submitted.

Conflicts of Interest: The authors declare no conflict of interest.

\section{References}

1. Layeghi, A.; Latifi, H.; Frazao, O. Magnetic Field Sensor Based on Nonadiabatic Tapered Optical Fiber with Magnetic Fluid. IEEE Photonics Technol. Lett. 2014, 26, 1904-1907. [CrossRef]

2. Huang, Y.; Li, D.; Li, F.; Zhu, Q.; Xie, Y. Transmitted light relaxation and microstructure evolution of ferrofluids under gradient magnetic fields. Opt. Commun. 2015, 338, 551-559. [CrossRef]

3. Agruzov, P.M.; Pleshakov, I.V.; Bibik, E.E.; Stepanov, S.I.; Shamrai, A.V. Transient magneto-optic effects in ferrofluid-filled microstructured fibers in pulsed magnetic field. Europhys. Lett. 2015, 111. [CrossRef]

4. Gao, R.; Jiang, Y.; Abdelaziz, S. All-fiber magnetic field sensors based on magnetic fluid-filled photonic crystal fibers. Opt. Lett. 2013, 38, 1539-1541. [CrossRef] [PubMed]

5. Shaohua, D.; Shengli, P.; Haotian, W. Magnetic field sensing based on magnetic-fluid-clad fiber-optic structure with taper-like and lateral-offset fusion splicing. Opt. Express 2014, 22, 19108-19116.

6. Wang, J.; Zhou, C.; Fan, D.; Ou, Y. Fiber optic magnetic field sensor based on magnetic fluid and etched Hi-Bi fiber loop mirror. In Proceedings of the OFS2014 23rd International Conference on Optical Fiber Sensors, Cantabria, Spain, 2-6 June 2014.

7. Homa, D.; Pickrell, G. Magnetic Sensing with Ferrofluid and Fiber Optic Connectors. Sensors 2014, 14, 3891-3896. [CrossRef] [PubMed]

8. Zhao, Y.; Lv, R.-Q.; Ying, Y.; Wang, Q. Hollow-core photonic crystal fiber fabry-perot sensor for magnetic field measurement based on magnetic fluid. Opt. Laser Technol. 2012, 44, 899-902. [CrossRef]

9. Candiani, A.; Argyros, A.; Leon-Saval, S.G.; Lwin, R.; Selleri, S.; Pissadakis, S. A loss-based, magnetic field sensor implemented in a ferrofluid infiltrated microstructured polymer optical fiber. Appl. Phys. Lett. 2014, 104, 111106.

10. Dai, J.; Yang, M.; Li, X.; Liu, H.; Tong, X. Magnetic field sensor based on magnetic fluid clad etched fiber Bragg grating. Opt. Fiber. Technol. 2011, 17, 210-213. [CrossRef]

11. Childs, P.; Candiani, A.; Pissadakis, S. Optical Fiber Cladding Ring Magnetic Field Sensor. IEEE Photonics Technol. Lett. 2011, 23, 929-931. [CrossRef] 
12. Chen, Y.; Han, Q.; Liu, T.; Lan, X.; Xiao, H. Optical fiber magnetic field sensor based on single-mode-multimode-single-mode structure and magnetic fluid. Opt. Lett. 2013, 38, 3999-4001. [CrossRef] [PubMed]

13. Lin, W.; Miao, Y.; Zhang, H.; Liu, B.; Liu, Y.; Song, B. Fiber-optic in-line magnetic field sensor based on the magnetic fluid and multimode interference effects. Appl. Phys. Lett. 2013, 103, 151101. [CrossRef]

14. Deng, M.; Sun, X.; Han, M.; Li, D. Compact magnetic-field sensor based on optical microfiber Michelson interferometer and $\mathrm{Fe}_{3} \mathrm{O}_{4}$ nanofluid. Appl. Opt. 2013, 52, 734-741. [CrossRef] [PubMed]

15. Zu, P.; Chan, C.C.; Koh, G.W.; Lew, W.S.; Jin, Y.; Liew, H.F.; Wong, W.C.; Dong, X. Enhancement of the sensitivity of magneto-optical fiber sensor by magnifying the birefringence of magnetic fluid film with Loyt-Sagnac interferometer. Sens. Actuators B. Chem. 2014, 191, 19-23. [CrossRef]

16. Zu, P.; Chan, C.C.; Lew, W.S.; Jin, Y.; Zhang, Y.; Liew, H. F.; Dong, X. Magneto-optical fiber sensor based on magnetic fluid. Opt. Lett. 2012, 37, 398-400. [CrossRef] [PubMed]

17. Zu, P.; Chan, C.C.; Lew, W.S.; Hu, L.; Jin, Y.; Liew, H.F.; Chen, L.H.; Wong, W.C.; Dong, X. Temperature-insensitive magnetic field sensor based on nanoparticle magnetic fluid and photonic crystal fiber. IEEE Photonics J. 2012, 4, 491-498.

18. Zu, P.; Chan, C.C.; Gong, T.; Jin, Y.; Wong, W.C.; Dong, X. Magneto optical fiber sensor based on band gap effect of photonic crystal fiber infiltrated with magnetic fluid. Appl. Phys. Lett. 2012, 101, 241118. [CrossRef]

19. Scarcia, W.; Palma, G.; Falconi, M.C.; de Leonardis, F.; Passaro, V.; Prudenzano, F. Electromagnetic Modelling of Fiber Sensors for Low-Cost and High Sensitivity Temperature Monitoring. Sensors 2015, 15, 29855-29870. [CrossRef] [PubMed]

20. Lv, R.Q.; Zhao, Y.; Wang, D.; Wang, Q. Magnetic Fluid-Filled Optical Fiber Fabry-Perot Sensor for Magnetic Field Measurement. IEEE Photonics Technol. Lett. 2014, 26, 217-219. [CrossRef]

21. Chen, Y.F.; Yang, S.Y.; Tse, W.S.; Horng, H.E.; Hong, C.Y.; Yang, H.C. Thermal effect on the field-dependent refractive index of the magnetic fluid film. Appl. Phys. Lett. 2003, 82, 3481-3483. [CrossRef]

(C) 2016 by the authors; licensee MDPI, Basel, Switzerland. This article is an open access article distributed under the terms and conditions of the Creative Commons Attribution (CC-BY) license (http://creativecommons.org/licenses/by/4.0/). 